\title{
A Novel Signal Scrambling Technique for PAPR Reduction in OFDM Systems
}

\author{
Anjana Sharma, Amarjeet Kaur, Shikha Saxena, Prashant Singh
}

\begin{abstract}
OFDM forms the basis of the upcoming next generation technologies so as to achieve higher data rates within a given bandwidth effectively. One of the major issues associated with OFDM is Peak to Average Power Ratio (PAPR) which needs to be minimized to get an efficient performance. The random variation in the signal amplitude of the OFDM signal leads to additional interference in the system and hence affecting the performance of HPA in non-linear region. In this paper, we propose a technique for the reduction of PAPR in OFDM systems with some increased complexity which works for any modulation type and any number of subcarriers. The simulation results show performance improvement with respect to the existing signal scrambling techniques.
\end{abstract}

Index Terms: Orthogonal frequency division multiplexing (OFDM), Peak to Average Power Ratio (PAPR), High Power Amplifier (HPA), Complimentary Cumulative Distribution Function (CCDF).

\section{INTRODUCTION}

OFDM converts frequency selective fading channels into narrowband flat fading channels which do not suffer from the problem of Inter Symbol Interference (ISI). Orthogonality among the subcarriers prevents subcarrier interference and effective utilization of the available bandwidth is achieved. OFDM is computationally efficient as it uses FFT techniques instead of bank of modulators and demodulators at the transmitter and receiver respectively[1]. Several advantages associated with OFDM includes higher spectral efficiency, high data rate[2] making it quite suitable for application in a number of modern world technologies like IEEE 802.11a, g, $\mathrm{j}, \mathrm{n}$ (WiFi) Wireless LANs, IEEE 802.15.3a Ultra Wideband (UWB) IEEE $802.16 \mathrm{~d}$, e (WiMAX), Wireless MANs, IEEE 802.20 Mobile Broadband Wireless Access (MBWA), DVB (Digital Video Broadcast) , 3GPP UMTS \& 3GPP LTE (Long-Term Evolution), 4G[3].

The problem of high PAPR arises in OFDM when different subcarriers combine constructively in same phase. The peak power of OFDM signal increases with the increase in number of subcarriers. HPA used for transmitting the OFDM signal at a higher power is generally operated near the saturation region but the non-linear characteristics of a signal may affect the efficiency of the HPA as it is quite sensitive to the random signal amplitude variation[4]. This may cause the inter modulation between the subcarriers thereby adding to the interference to the system which is quite undesirable. High PAPR also requires a high precision DAC to be deployed which has a very high cost wherein a normal DAC with low dynamic range would offer low signal to quantization noise ratio for a given sampling rate[5]. A number of PAPR reduction techniques have been proposed in the recent past which include clipping[6], windowing[7], tone reservation (TR)[8], tone interjection (TI)[8], active constellation extension (ACE)[9], signal scrambling techniques like partial transmit sequence (PTS)[10],[11], selective mapping(SLM)[12],[13] and interleaving[13].

In this paper, we propose a technique for PAPR reduction in OFDM which optimizes the PAPR by multiplying the sub blocks of modulated data with different weight factor vectors, taking their inverse Fourier transform, selecting the best weight factor vector which gives the least PAPR and combining them to get an optimized OFDM signal having low PAPR. The performance of HPA along with receiver detection efficiency would also be satisfactory for different modulation schemes and any number of subcarriers.

\section{OFDM FUNDAMENTALS}

Orthogonal frequency division multiplexing is a high data rate parallel transmission scheme in which different symbols are modulated onto the different orthogonal subcarriers [1]. As shown below:

$$
s(t)=\sum_{i} X_{i} e^{j 2 \pi i \frac{B}{N} t}
$$

Where $s(t)$ represents the composite signal, $\quad j=\sqrt{-1}, B$ is the total bandwidth allocated to the system, $\frac{B}{N}$ is the subcarrier bandwidth and trepresents the time instant.

We take the sampled signal and the samples are nothing but the DFT of the information symbols. So, in OFDM, we have a very efficient way to generate the samples.

Considering $u^{\text {th }}$ sample

$$
\begin{aligned}
& t=u T_{s}=\frac{u}{B} \\
& s\left(u T_{s}\right)=\sum_{i} X_{i} e^{j 2 \pi i \frac{u}{N}}
\end{aligned}
$$

Revised Manuscript Received on June 15, 2019.

Anjana Sharma, Amarjeet Kaur, Shikha Saxena and Prashant Singh

: CGC Landran, Mohali, Punjab, India 


\section{A Novel Signal Scrambling Technique for PAPR Reduction in OFDM Systems}

Where $T_{s}$ defines the sampling period, and (3) gives us DFT of the information symbols.

The main advantage of using this multicarrier modulation is that the whole bandwidth gets divided into several number of smaller bandwidth subcarriers $(B / N)$ resulting in frequency flat fading instead of the frequency selective fading experienced by the single carrier modulation schemes. The single carrier modulation schemes transmit one symbol in one time period offering an effective data rate equal to the bandwidth of the system. Multicarrier modulation schemes also have the same data rate as that of single carrier modulation as they transmit $N$ symbols in $N$ time periods but there is no Inter Symbol Interference (ISI) present[2].

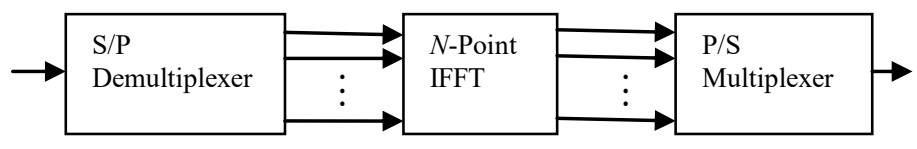

Transmitter

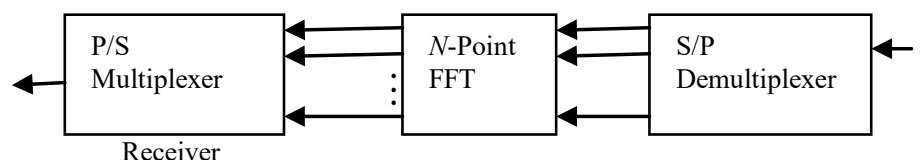

Figure 1. OFDM Transmitter and Receiver Schematic with $N$ number of subcarriers.

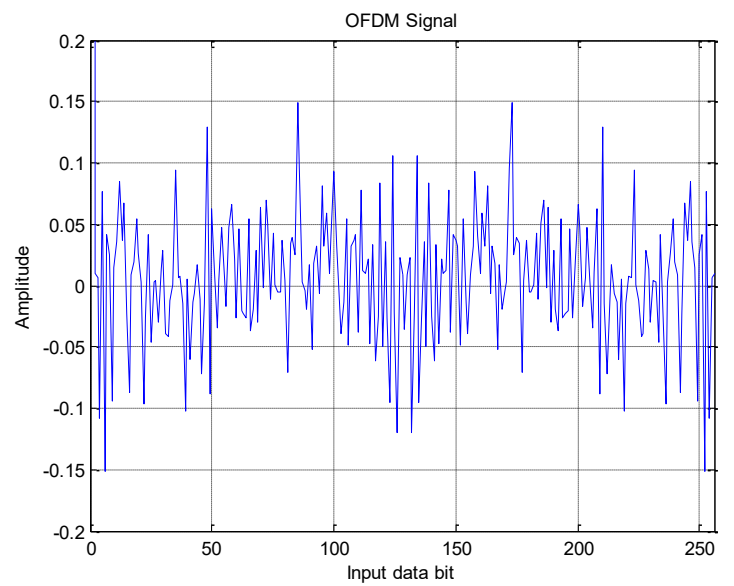

Figure 2. QPSK modulated OFDM signal for 256 bit long sequence.

\section{PAPR IN OFDM}

The signal to be transmitted through the channel in a multicarrier modulation scheme like OFDM is the sum of several message signals modulated upon different orthogonal subcarriers having equal bandwidth. When these signals combine with same phase at a point the peak power is increased. PAPR may be defined as the ratio of peak power to the average power for a given signal where peak power may be characterized as the power of a sine wave having an amplitude equal to the maximum envelope value of the signal[3]. In OFDM, we do not transmit the information symbols but the IFFT samples of the same. If $x(k)$ is the $k^{\text {th }}$ IFFT sample given as:

$$
x(k)=\frac{1}{N} \sum_{i=0}^{N-1} X(i) e^{j 2 \pi k i / N}
$$

Where $X(i)$ represents $i^{\text {th }}$ information symbol and $N$ is the number

$$
P A P R=\frac{\max |x(k)|^{2}}{E\left[|x(k)|^{2}\right]}
$$

From (4) and (5) we may infer that more the number of subcarriers, more will be the PAPR.

\section{A. Problems due to PAPR}

Major motivation behind reducing the PAPR in an OFDM system is the performance of HPA in the nonlinear region[4]. It is quite sensitive to the random amplitude variations which further results in interference due to inter modulation between the subcarriers.

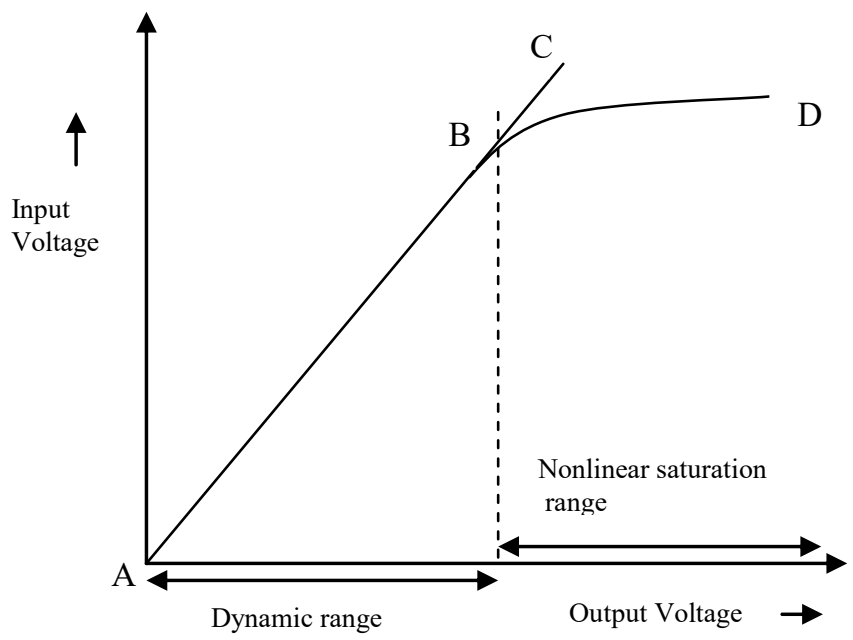

Figure 3. High Power Amplifier characteristics

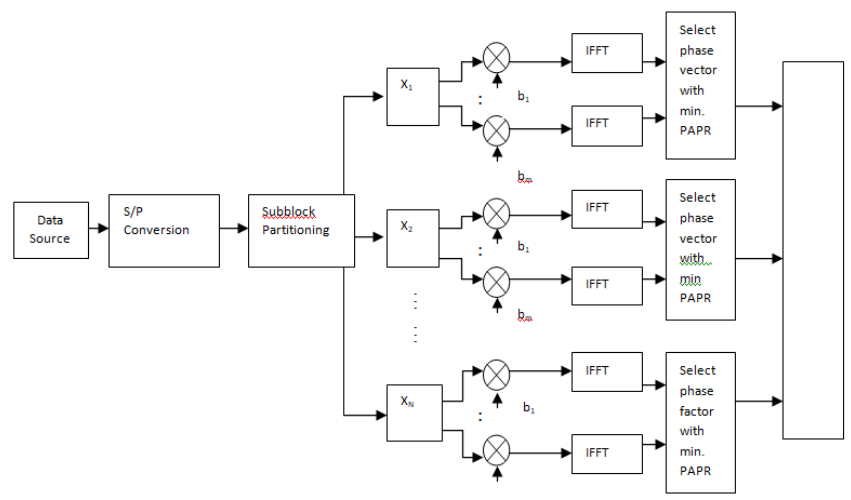

Figure 4. Block diagram of the proposed technique

Figure. 3 shows the performance of HPA corresponding to the different regions of operation. When the peak deviation about the average is significantly high, the signal level moves outside the dynamic range i.e A to B. High PAPR results in amplifier saturation thus leading to nonlinearity and hence loss of orthogonality among the subcarriers i.e the amplifier's region of operation moves towards $\mathrm{D}$ which is quite undesirable[4].

To deal with high peak values, DACs with high dynamic range are needed. Low precision DACs offer high quantization noise when the dynamic range is increased and on the other hand, high 
precision amplifiers are quite costly.

\section{A. Existing techniques of PAPR reduction}

Several techniques have been proposed by the researchers to reduce the PAPR of an OFDM signal. Some of them are listed below[6-12]:

1) Coding: It works on the principle of choosing the codewords with minimum PAPR and transmitting them. This technique suffers from exhaustive search for the codewords and it does not provide with error correcting.

2) Amplitude clipping and filtering: Clipping the amplitude of the OFDM symbol also can be used as a technique for PAPR reduction. This suffers from in-band and out of band radiation.

3) Signal scrambling: Partial transmit sequence and selected mapping are the two techniques which are quite effective when it comes to reduction of PAPR in OFDM systems.

\section{PROPOSED WORK}

In this technique, we consider an input data block of $\mathrm{M}$ bits which is further divided into $\mathrm{N}$ number of uncorrelated subblocks. Each subblock is oversampled by L so as to have data length equal to that of the original input data stream. Each subblock is multiplied by the available set of phase factors vector. IDFT of the available vectors is taken and PAPR is calculated for each. Phase factor vector which gives the least PAPR is chosen and selected for the corresponding subblock. Adding up all the subblocks with optimal phase factor vectors for each correspondingly will give us the signal to be transmitted as shown in the fig 4 . The data block $X$ is divided into subblocks of equal length such that $X=\sum_{n=1}^{N} X_{n}$

where $n=1,2,3 \ldots \mathrm{N}$ are the subblocks which after being optimized with the phase factor vectors and IFFT of which are added to minimize the PAPR in time domain. The phase factor vectors are given by $b_{m}$, for the simulation purposes we have taken the value of $m=4$.The length of $b_{m}$ is equal to the length of the input bit sequence having values from the set $S=\left\{e^{j 2 \pi l / W} \mid l=1,2 \ldots . W-1\right\}[11-13]$, where $\mathrm{W}$ is the number of allowed phase factors which we have taken as 4 here. The amount of PAPR reduction using this technique is quite impressive with a little increase in the complexity of the algorithm because of the deployment of IFFT blocks for every subblock optimized with the phase factor vector. Also, the phase factor vectors don't need to have a value assigned to every bit in the vector as we have oversampled the signal by an amount $\mathrm{L}$.

The elements of the subblock which have a value assigned from the set of corresponding input sequence are meant to be optimized with the phase factor vector and hence we may control the complexity by reducing the number of phase factor by an amount of $(N-1) / N$. At the receiver, the information of these phase factor vectors is needed to recover the optimized OFDM signal back in its original form for the applications to process it. The proposed technique works for any modulation scheme and any number of subcarriers with an acceptable amount of delay because of the IFFT blocks involved.

\section{SIMULATION RESULTS}

The simulation parameters taken into consideration for simulating the proposed system are given in the Table 1 .

TABLE 1. Simulation Parameters

\begin{tabular}{|l|l|}
\hline Parameters & Values \\
\hline Bit sequence length & 256 \\
\hline Modulation & QPSK \\
\hline Subblock partitioning & Adjacent partitioning \\
\hline Number of subblocks & 4 \\
\hline Number of phase factors allowed & $4(1,-1, \mathrm{j},-\mathrm{j})$ \\
\hline Number of phase factor vectors & 4 \\
\hline IFFT length & 256 \\
\hline
\end{tabular}

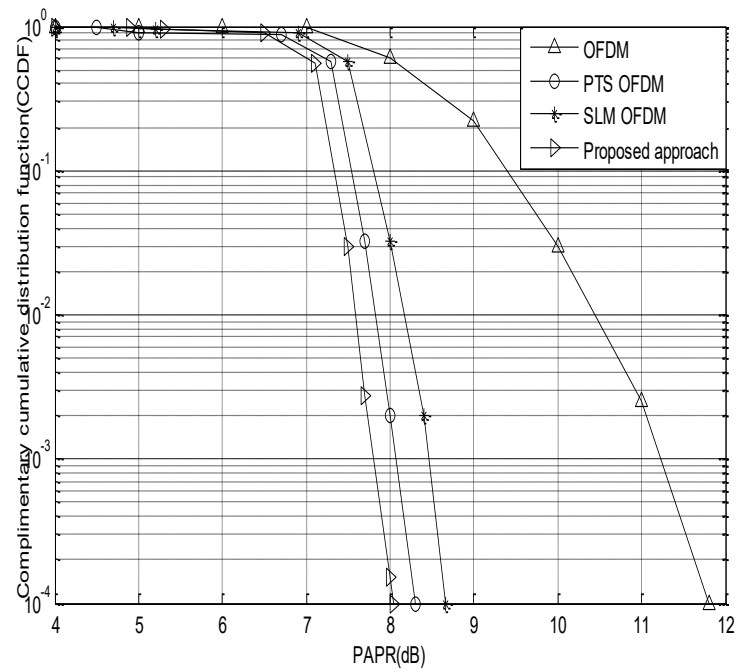

Figure 5. CCDF of an OFDM signal with 256 subcarriers compared with SLM and PTS.

Figure 5. shows that the probability of PAPR being less than $8.1 \mathrm{~dB}$ for SLM, PTS and the proposed approach is $0.0022,0.0019$ and 0.0001 respectively. As the number of subcarriers increase, the probability of PAPR being less than a given threshold decreases and hence the performance degrades.

\section{CONCLUSION}

In this paper, we studied and analyzed the results of novel technique for PAPR reduction in OFDM system which has a CCDF performance better than that of the existing signal scrambling techniques like PTS and SLM. The increase in complexity of the technique is because of the increased number of IFFT blocks is acceptable for the amount of PAPR reduction it offers. In the coming future we will try to implement this technique in MIMO-OFDM. 


\section{AUTHORS PROFILE}

Ms. Anjana Sharma is assistant Professor in Chandigarh Engineering College since 2014. She has 7+ years experience in teaching and has more than 13 papers in international journals and international conferences.

Ms. Amarjeet Kaur is assistant Professor in Chandigarh Engineering College since 2014. She has $10+$ years experience in teaching and has more than 9 papers in international journals and international conferences.

Dr. Shikha Saxena is assistant Professor in Chandigarh Engineering College since 2018. She has $8+$ years experience in teaching and has more than 10 papers in international journals and international conferences. 